\title{
Microvolume trace environmental analysis using peak-focusing online solid-phase extraction-nano-liquid chromatography-high-resolution mass spectrometry
}

\author{
Michael A. Stravs ${ }^{1,2} \cdot$ Jonas Mechelke $^{1} \cdot$ P. Lee Ferguson ${ }^{3} \cdot$ \\ Heinz Singer ${ }^{1}$ • Juliane Hollender ${ }^{1,2}$
}

Received: 21 September 2015 / Revised: 18 December 2015 / Accepted: 22 December 2015 /Published online: 20 January 2016

(C) Springer-Verlag Berlin Heidelberg 2016

\begin{abstract}
Online solid-phase extraction was combined with nano-liquid chromatography coupled to high-resolution mass spectrometry (HRMS) for the analysis of micropollutants in environmental samples from small volumes. The method was validated in surface water, Microcystis aeruginosa cell lysate, and spent Microcystis growth medium. For 41 analytes, quantification limits of $0.1-28 \mathrm{ng} / \mathrm{L}$ (surface water) and $0.1-32 \mathrm{ng} /$ $\mathrm{L}$ (growth medium) were obtained from only $88 \mu \mathrm{L}$ of sample. In cell lysate, quantification limits ranged from $0.1-143 \mathrm{ng} / \mathrm{L}$ or $0.33-476 \mathrm{ng} / \mathrm{g}$ dry weight from a sample of $88 \mu \mathrm{L}$, or $26 \mu \mathrm{g}$ dry weight, respectively. The method matches the sensitivity of established online and offline solid-phase extraction-liquid chromatography-mass spectrometry methods but requires only a fraction of the sample used by those techniques, and is among the first applications of nano-LC-MS for environmental analysis. The method was applied to the determination of bioconcentration in Microcystis aeruginosa in a laboratory experiment, and the benefit of coupling to HRMS was demonstrated in a transformation product screening.
\end{abstract}

Electronic supplementary material The online version of this article (doi:10.1007/s00216-015-9294-x) contains supplementary material, which is available to authorized users.

Juliane Hollender

juliane.hollender@eawag.ch

1 Eawag, Swiss Federal Institute of Aquatic Science and Technology, 8600 Dübendorf, Switzerland

2 Institute of Biogeochemistry and Pollutant Dynamics, ETH Zurich, 8092 Zürich, Switzerland

3 Department of Civil and Environmental Engineering, Duke University, Durham, NC 27708, USA
Keywords Nano-LC · Environmental analytics · High-resolution MS · Miniaturization · Online SPE · Biological samples

\section{Introduction}

Organic micropollutants in the environment are a central topic of research in environmental analytical chemistry [1, 2]. While classical hydrophobic pollutants have been analyzed with gas chromatography-mass spectrometry (GC-MS) methods, the advent of electrospray ionization (ESI) [3] for the hyphenation of liquid chromatography (LC) with MS has made more hydrophilic micropollutants accessible to mass spectrometric analysis. In combination with sample enrichment techniques, highly sensitive detection can be achieved. Solid-phase extraction (SPE) followed by LC-MS is routinely applied for the analysis of micropollutants in water samples and biota. State-of-the-art methods are able to quantify a wide variety of micropollutants at low nanogram per liter or nanogram per gram levels [4-7]. To increase sample throughput, automated SPE can be employed, where enrichment on a cartridge is performed by the chromatographic system and elution is performed directly onto the chromatographic column (online SPE) [8]. In addition to automating otherwise tedious manual work, online SPE offers higher reproducibility and precision by reducing sample manipulation [9] and is much faster. While in manual (offline) SPE often L quantities of sample are enriched, online SPE can reach $\mathrm{ng} / \mathrm{L}$ detection limits from sample volumes of typically 1 to $20 \mathrm{~mL}$ [10-13] and the technique can be applied to biological material [14] where sample volume is often limited. In the most common setup, after enrichment, the SPE cartridge is switched in line with the analytical column and a gradient elution is performed over both (trap-and-elute). A key challenge in this setup is the 
choice of an SPE sorbent compatible with the analytical column while preventing analyte breakthrough [15]. A more advanced setup involves dilution of the SPE eluate before the analytical column $[10,16]$. This causes refocusing of the analytes on the analytical column and improves multiresidue analytical separation for analytes with a broad range of properties.

Advances in LC instrumentation have enabled the miniaturization of analytical methods. Capillary and nano-LC-MS provide the advantage of high sensitivity with reduced sample volumes, while separation is as efficient as in regular systems. As an additional benefit, solvent usage and waste generation is reduced [17]. Nano-LC typically refers to chromatography at sub-microliter per minute flow rates with capillary columns of up to $150 \mu \mathrm{m}$ inner diameter (ID) [18]. Since the chromatographic dilution scales with the square of the ID [19], a reduction of e.g., column diameter from $2.1 \mathrm{~mm}$ (a typical narrowbore column) to $100 \mu \mathrm{m}$ (a typical nano-LC column) would lead to a 441 -fold increase in mass sensitivity. Since the column capacity, and therefore maximum injectable sample amount, is also subject to scaling, nano-LC itself does not inherently confer higher sensitivity except in cases where sample volume is limited (e.g. for biological samples) [19]. However, hyphenation to mass spectrometry with micro- or nanoelectrospray also contributes to enhanced sensitivity, since the smaller emitted initial droplet size leads to more efficient desolvation and thus higher transmittance [20]. In nano-LC setups, the realization of complex setups such as column-switching SPE is dependent on careful consideration of geometry to avoid dead volumes which can impair separation efficiency [21]. Column-switching SPE in nano-LC systems has been realized for proteomics applications [22], or in hyphenation with ICP-MS for the analysis of lanthanidelabeled peptides [23]. However, to our knowledge, no SPE system incorporating pre-column dilution/peak refocusing has yet been described. Nano-LC-MS has gained widespread adoption in the field of proteomics [24], where small sample amounts are an inherent limitation. In environmental analysis, only few applications have been reported. In an early application, Wilson et al. combined online solid-phase extraction with nano-LC-MS for the analysis of perflurooctanoic acid and perfluorooctane sulfate, reaching method detection limits of 0.5 and $1 \mathrm{pg}$, respectively [25]. Recently, interest in miniaturized applications for multiresidue environmental analytics has resurged. Berlioz-Barbier et al. used miniaturized QuEChERS extraction followed by nano-LC-MS for the analysis of carbamazepine and fluoxetine in benthic invertebrates [26]; a similar setup was applied for 35 micropollutants [27]. David et al. developed a miniaturized extraction on multi-well plates in combination with nano-UHPLC-MS for profiling of the metabolome and xenometabolome in fish plasma, which includes environmentally relevant substances [28]. In a related study, Chetwynd et al. showed a sensitivity increase for metabolites and xenometabolites using nano-LC-nano-ESIMS [29]. Nano-LC was also applied as the first separation dimension in a 2D-LC system with ESI-MS detection for comprehensive screening of a wastewater sample [30]. However, no multiresidue method to date combines nanoLC-MS with automated online SPE, despite the fact that this avoids problematic manual handling of extremely small volumes, and offers automatization and improved reproducibility.

Our aim was to develop a system which could be used for highly sensitive analysis of organic micropollutants in small sample quantities, such as phytoplankton samples from surface water or high-throughput laboratory experiments (e.g. in multi-well plates). To develop a method applicable for a broad range of analytes with very small volumes, we aimed to implement refocusing online SPE combined with nano-LC-MS. Using 59 environmentally relevant substances with a broad range of polarity (octanol-water partitioning coefficient (log Dow) values at $\mathrm{pH} 7$ of -2.1 to 4.8 ) and molecular weight (119 to $748 \mathrm{Da}$ ), the apparatus configuration and analytical method was developed, optimized and finally the capabilities and limitations of the method evaluated. The use of high-resolution mass spectrometry enabled the application not only to multiresidue analysis but also for the identification of transformation products via suspect screening [31].

\section{Materials and methods}

Apparatus The chromatographic system consisted of a Dionex UltiMate TM 3000 RSLCnano (Thermo Scientific, Bremen) with an NCS-3500RS pump module and a WPS3000 TPS RS autosampler, and a Rheos 2200 quaternary HPLC pump (Flux Instruments, Switzerland) used as an auxiliary pump. The pump module contains a binary micro/nano pump which was equipped with a nano-LC flow selector, and a ternary loading pump. The solvent channels of the loading pump were routed through the built-in four-channel degasser of the chromatographic system. An additional degasser with reduced chamber volume (DEGASi micro, 2 channels, Biotech, Sweden) was used to degas the nano-LC solvent channels. In addition to the 10-port/2-position switching valve installed in the column compartment, a second 10-port-/2-position switching valve (model C72X-6670D) was operated externally with a microelectric actuator (model ED, both VICI, Schenkon, Switzerland) controlled via relay from the chromatographic system. Online solid-phase extraction was performed using EXP Stem Trap cartridges with an inner diameter of $130 \mu \mathrm{m}$ and a volume of $170 \mathrm{~nL}$ (Optimize Technologies, USA) custom-packed using tools provided by the manufacturer. The cartridges were packed with Oasis HLB (Waters, USA), PolyWAX LP (PolyLC, USA), PolyCAT A (PolyLC, USA) or mixtures thereof, in $5 \mu \mathrm{m}$ particle size. Chromatography was performed over an Atlantis dc 18 
nanoACQUITY column (Waters, USA; $100 \AA$, $3 \mu \mathrm{m}$, $100 \mu \mathrm{m} \times 150 \mathrm{~mm}$ ). The chromatographic system was coupled to a Q-Exactive Plus (Thermo Scientific, Bremen) single quadrupole-orbitrap mass spectrometer using a Nanospray Flex (Thermo Scientific, Bremen) ion source, with a modified junction for applying high voltage. The ion source was equipped with a TaperTip emitter (New Objective, USA; $360 \mu \mathrm{m}$ OD, $50 \mu \mathrm{m} \mathrm{ID}$, uncoated) cut to approximately $5 \mathrm{~cm}$ length and voltage was applied at the junction between column outlet and emitter. All connections between components were made with either nanoViper PEEK-coated fused-silica capillaries with built-in zero-dead-volume 1/16" fittings (Thermo Scientific, Bremen) or fused-silica capillaries (360 $\mu \mathrm{m}$ OD, BGB, Switzerland) with two-piece PEEK $360 \mu \mathrm{m}$ to $1 / 16$ " adaptor fittings (VICI, Schenkon, Switzerland). Connections configured for nanoflow rates generally used $20 \mu \mathrm{m}$ ID capillaries, whereas connections for higher flow rates generally used $75 \mu \mathrm{m}$ capillaries. A detailed description of the connections is found in Fig. S1 and Table S1 in the Electronic Supplementary Material (ESM).

Solvents The binary nano-LC pump delivered nanopure water with $0.1 \%$ formic acid (solvent A) and LC-MS grade methanol with $0.1 \%$ formic acid (solvent B). The auxiliary pump supplied solvent A isocratically and the ternary loading pump $5 \mathrm{mM}$ ammonium acetate in nanopure water (loading solvent), LC-MS grade acetonitrile, and 10\%/90\% LC-MS grade methanol/nanopure water (solvent C). All solvents were degassed for $30 \mathrm{~min}$ in an ultrasonic bath and filtered through $0.2 \mu \mathrm{m}$ regenerated cellulose membrane filters (Sartorius, Germany). Solvent $\mathrm{C}$ was used for the syringe buffer of the autosampler.

Online SPE-nano-LC setup The configuration of the onlineSPE-nano-LC coupling is shown in a summary in Fig. 1 and in more detail in ESM Fig. S2. A chromatographic run started with a loading step, in which a sample was loaded and concentrated on the SPE cartridge. This was followed by an elution step, where the concentrated sample was eluted from the SPE cartridge and refocused on the chromatographic column. Finally, in the chromatographic step, gradient chromatography over the column took place. The 10-port valve connected to the chromatographic column (the method valve) assumed two positions. Position A was used for the loading and chromatography steps, whereas during elution the valve was switched to position B.

The gradient program and valve positions are listed in Table 1. During loading, the loading pump delivered a $10 \mu \mathrm{L} / \mathrm{min}$ flow of $98 \%$ loading solvent $/ 2 \%$ acetonitrile to the SPE cartridge. The autosampler drew $44 \mu \mathrm{L}$ sample into the sample loop and then switches it into the loading pump flow. The flow then delivered the sample from the sample loop to the SPE cartridge during $\sim 5 \mathrm{~min}$. Subsequently, the process was repeated, such that finally $88 \mu \mathrm{L}$ sample were concentrated on the SPE cartridge during $\sim 10 \mathrm{~min}$. The loading process was performed using a custom autosampler program to minimize cross-contamination (see SI for details). During this time, the nano-LC pump conditioned the analytical column to $10 \% \mathrm{~B}$ at a flow of $700 \mathrm{~nL} / \mathrm{min}$. The auxiliary pump delivered a flow of $900 \mathrm{~nL} / \mathrm{min}$ of A against a flow restrictor capillary, which serves to provide backpressure for the pump to ensure a constant flow rate.

After the second sample plug had passed the SPE cartridge, the nano-LC pump lowered the flow rate to $120 \mathrm{~nL} / \mathrm{min}$ while simultaneously changing the solvent to $95 \% \mathrm{~B}$, and the method valve switched to position $B$. In this position, the flow over the column was composed from $900 \mathrm{~nL} / \mathrm{min}$ A from the auxiliary pump and $120 \mathrm{~nL} / \mathrm{min} 95 \% \mathrm{~B}$ cartridge eluate from the nano-LC pump. The total elution time was $9.5 \mathrm{~min}$. While still in elution position, the nano-LC pump switched back to aqueous $(10 \% \mathrm{~B})$ conditions.

Subsequently, the chromatographic step was initiated. The valve switched back to position A and flow was quickly raised to $700 \mathrm{~nL} / \mathrm{min}$. The nano-LC pump delivered a chromatographic gradient of $1.8 \mathrm{~min}$ at $10 \% \mathrm{~B}, 6 \mathrm{~min}$ from $10 \%$ to $50 \% \mathrm{~B}, 11.8 \mathrm{~min}$ from $50 \%$ to $95 \% \mathrm{~B}, 3.5 \mathrm{~min}$ at $95 \% \mathrm{~B}$, $0.5 \min 95 \%$ to $10 \% \mathrm{~B}$.

During the chromatography step, the loading pump solvent was switched to acetonitrile, and the SPE cartridge was washed for $9.3 \mathrm{~min}$ after the chromatography step started. Subsequently, the solvent was changed back to $98 \%$ loading solvent $/ 2 \%$ acetonitrile, and the cartridge was reequilibrated for 9 min until the end of the run. Due to a $200-\mu \mathrm{L}$ gradient delay of the loading pump, purge steps were incorporated for every solvent change using an additional valve (see Table 1 for details).

Nano-ESI and detection The use of commercial nanoelectrospray Silica TaperTip emitters was important for achieving good spray conditions reproducibly over multiple months. Stainless steel emitters were also tested, but found to give less reproducible spray conditions and a less stable spray over the chromatographic gradient. Positive mode electrospray mass spectrometry was performed in full scan MS1 with top 3 data dependent MS2 using an inclusion list with the exact masses of analyte ions. The MS1 scan was performed at a resolution of 70,000 with a scan range of 100 to $1500 \mathrm{~m} / \mathrm{z}$ with a maximum injection time of $50 \mathrm{~ms}$. The MS2 scans were performed with an isolation window of $1.5 \mathrm{~m} / \mathrm{z}$ at a resolution of 17,500 with an automatically determined scan range and maximum injection time of $50 \mathrm{~ms}$. Collision energies for the analytes (see ESM Table S6) were determined using an empirical formula based on molecular weight and adjusted where necessary. When no inclusion list ions were found, top 3 precursor ions were fragmented with a collision energy setting of 50 (NCE, normalized collision 

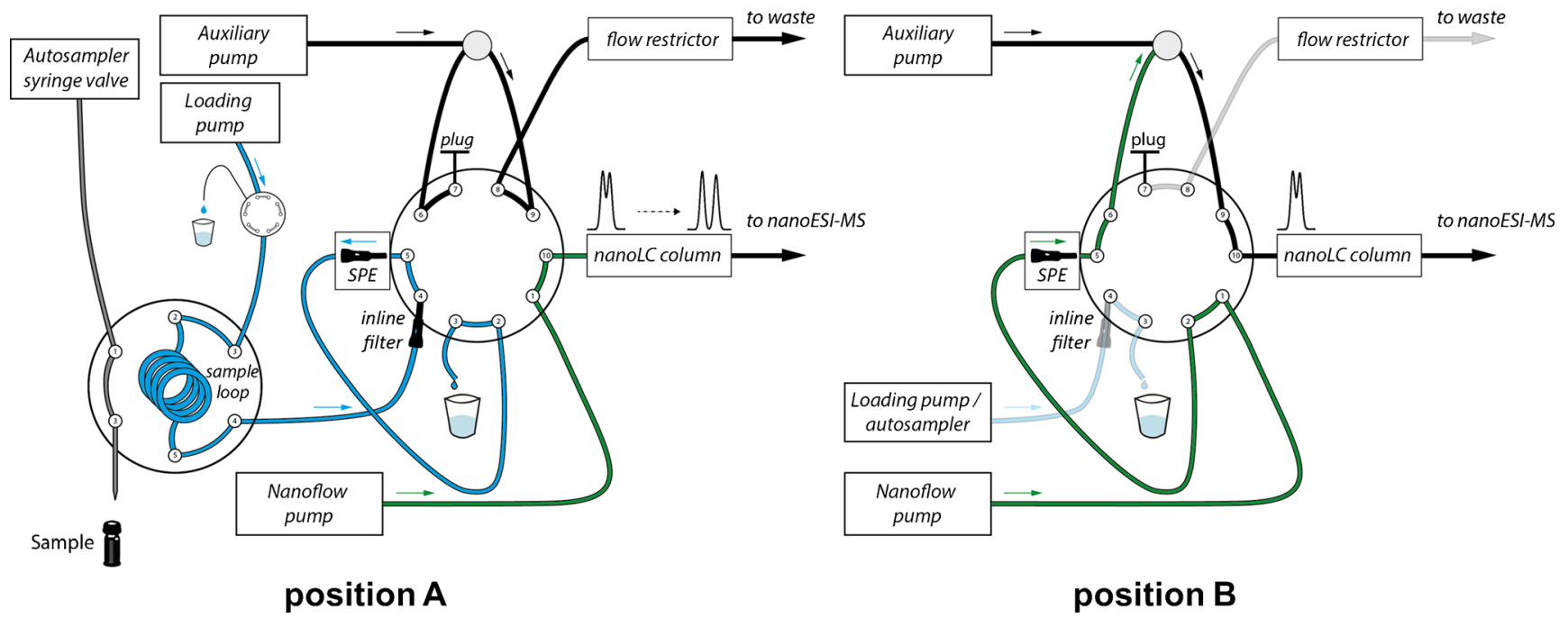

Fig. 1 Valve configuration of the final online-SPE-nano-LC setup. Left Valve position A, SPE loading (step 1) and chromatography (step 3). The loading pump delivers the sample loop contents to the SPE cartridge, while the nano-LC pump is connected directly to the column. Right Valve

position B, elution of SPE cartridge to column (step 2). The nano-LC pump delivers a low flow over the SPE cartridge to elute the analytes, which is diluted by the auxiliary pump flow before it reaches the column (more details in ESM Fig. S2). See Table 1 for the corresponding program

Table 1 Gradient and valve program of the analytical method. Method valve positions refer to the positions shown in Figure 1

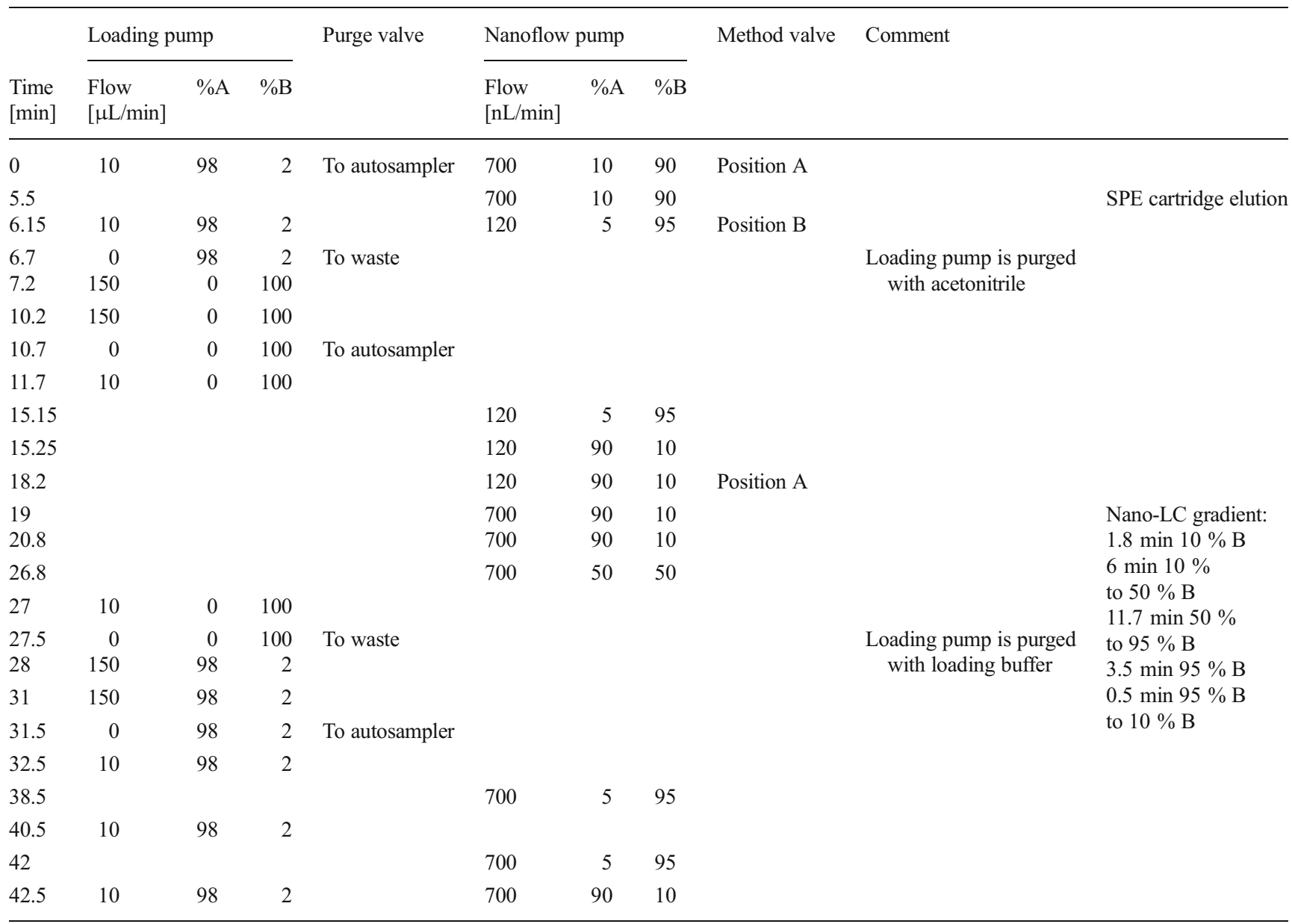

Solvent A: nanopure water with $0.1 \%$ formic acid, solvent B: methanol with $0.1 \%$ formic acid 
energy). Dynamic exclusion was set to $15 \mathrm{~s}$. Spray voltage was set at $2200 \mathrm{~V}$. No sheath, sweep, and auxiliary gas flows were used.

Data processing Quantification of analytes using the internal standard method was performed with TraceFinder EFS (version 3.2 RC, Thermo Scientific, Bremen). A mass tolerance of $5 \mathrm{ppm}$ was used. Analyte peaks were automatically integrated by the ICIS algorithm and reviewed by hand. Confirming fragments (see ESM Table S6) were automatically detected. Calibration curves were weighted $1 / x$ over the concentration range.

Sample collection Surface water (SW) was collected at Greifensee, Switzerland, at a depth of $2 \mathrm{~m}$, and stored at $4{ }^{\circ} \mathrm{C}$ until usage. For Microcystis cell lysate (MC) and spent Microcystis growth medium (GM), cells of Microcystis aeruginosa PCC7806 were grown in WC medium (see SI) to a concentration of $0.3 \mathrm{~g} / \mathrm{L}$ as determined via correlation to optical density. Twenty milliliters of the culture was centrifuged at $4000 \mathrm{rpm}$ for $10 \mathrm{~min}$. Supernatant was recovered as GM matrix. Remaining supernatant was removed. Cells were resuspended in $2 \mathrm{~mL}$ of nanopure water and cells were lysed by three cycles of freezing in liquid nitrogen and thawing at $37^{\circ} \mathrm{C}$ in an ultrasonic bath. The suspension was then frozen and freeze-dried. The resulting pellet was resuspended in $4 \mathrm{~mL} \mathrm{1:1} \mathrm{ethanol:nanopure} \mathrm{water} \mathrm{and} \mathrm{incubated} \mathrm{for} 10 \mathrm{~min}$ in an ultrasonic bath at $37{ }^{\circ} \mathrm{C}$. The suspension was centrifuged and the supernatant was diluted 1:10 with nanopure water, giving $\mathrm{MC}$ matrix.

Sample preparation Matrix samples were filtered through $0.2 \mu \mathrm{m}$ regenerated cellulose syringe filters $(0.22 \mu \mathrm{m}$, $25 \mathrm{~mm}, \mathrm{BGB}$, Switzerland). To every sample of $1500 \mu \mathrm{L}$, $30 \mu \mathrm{L} 0.1 \mathrm{M}$ ammonium citrate buffer $(\mathrm{pH} 7)$ was added. For quantification, $30 \mu \mathrm{L}$ of a mixture of isotope-labeled internal standards (ILIS; see ESM Table S4) was added to a final concentration of $20 \mathrm{ng} / \mathrm{L}$.

Method validation The performance of the analytical method was validated in nanopure water (NPW) and in three matrices: SW, GM, and MC. Parameters determined were limits of quantification (LOQ) for HRMS alone and with a confirming fragment, precision, accuracy, matrix effects, carryover and absolute extraction recovery (ER). Samples were fortified with a mixture of compounds at different concentration levels. From an initial selection of 59 compounds (see ESM Table S3), 39 were selected for validation. Twenty-two substances were quantified with matching ILIS (e.g., venlafaxine$d 6$ for the quantification of venlafaxine), while for the remaining 17 substances a standard at a similar retention time was selected. Calibration curves were determined from NPW samples fortified at 10 concentration levels $(0.1,0.2,0.5,1,2,5$,
20, 50, 100, $500 \mathrm{ng} / \mathrm{L}$ ). Matrix blank (no internal standard and no fortification) and method blank (internal standard but no fortification) samples were used to determine background levels or the absence thereof. All measurements were made in triplicate. LOQ was determined in NPW as the lowest concentration at which a peak with at least five measurement points was observed whose signal-to-noise ratio exceeded 10 , and the integrated area was at least $2 \times$ the matrix blank value. For SW, GM, and MC matrices, the matrix factor was calculated as the suppression or enhancement of peak areas relative to NPW for the corresponding ILIS (where available) or, respectively, peak areas of the analytes (where no matching ILIS was available). Corresponding LOQs in SW, GM, and $\mathrm{MC}$ were derived from the matrix factors. Carryover was determined by running a NPW blank sample after injections. For the determination of absolute extraction recovery, samples without added ILIS were injected and the eluate from the elution step was diverted to a collection vial instead of the column. ILIS was added to the eluates and the resulting samples measured with the regular method. Standard mixture was either added (a) to the matrix samples before injection, (b) to the eluates at a substance amount corresponding to $100 \%$ recovery, or (c) not at all (blank).

\section{Results and discussion}

Method development The online-SPE-nano-LC-ESI system was constructed to ensure both a reasonably easy handling and high reproducibility. The implemented refocusing approach was crucial to obtain good analytical separation. In conventional trap-and-elute setups, during the elution/ chromatography phase the enrichment cartridge and column will be in line (basically forming a prolonged column together) and the gradient runs over cartridge and column simultaneously. This setup is simple and convenient, but limits the selection of the SPE sorbent. If a strong SPE sorbent is chosen, with the goal to retain a wide range of analytes, it will often be the case that analytes are eluted from the SPE cartridge late, and are not well separated on the analytical column. Conversely, if the chosen sorbent is weak, analytes weakly retained in the sorbent material can be flushed out because of the high flow during loading. Early experiments with such a setup showed the former case, which resulted in broad peak shapes especially for early-eluting analytes and resulted in isobaric species not being separated (Fig. 2, left). It is noteworthy that analyte behavior in the trap-and-elute setup is not strictly correlated with retention time. Verapamil was an example of an early-eluting analyte well-behaved in trap-andelute mode. Verapamil was quickly eluted from the SPE cartridge and therefore well separated during chromatography. On the other hand, D617, venlafaxine and the didesmethylvenlafaxine metabolites were relatively strongly 
retained by the SPE cartridge and therefore were not well resolved on the analytical column subsequently. Ideally, for a trap-and-elute setup, a combination of SPE cartridge and analytical column should be found which results in good separation of all analytes of interest. However, with increasing number of analytes, it becomes increasingly difficult to find a combination suitable for every substance. In the refocusing setup, the cartridge was eluted with $95 \%$ organic phase such that elution was exhaustive and fast, and the eluate was diluted online pre-column with nanopure water such that the analytes were focused on the analytical column. This effectively separated SPE elution from chromatography, and resulted in resolution of the didesmethylvenlafaxine isobars and good peak shapes for venlafaxine and D617 verapamil metabolite (Fig. 2, right). At the same time, previously well-resolved peaks remained unaffected. Refocusing has been shown to be important for good analytical separation over a wide range of compounds in narrowbore and analytical online-SPE-LCMS systems [10, 16, 32]. The realization of dilution/ refocusing presents particular challenges in nanoflow regimes: During elution, the organic solvent delivered by the elution flow should not exceed $\sim 10 \%$ of the aqueous dilution flow, to successfully achieve refocusing of the analytes on the column, while the total flow is limited by the pump and column backpressure. These limiting factors dictate the use of a very low elution flow $(120 \mathrm{~nL} / \mathrm{min})$, which leads to a tradeoff between the elution volume through the SPE cartridge and prolonged elution times. Since the elution flow is one order of magnitude lower than the total flow $(1.02 \mu \mathrm{L} / \mathrm{min})$, considerations of dead volume, e.g., in junctions and capillaries become even more critical. Through the use of a conventional HPLC pump (Rheos 2200) for the dilution flow, a refocusing setup could be realized cost-effectively, avoiding the use of an additional nano-LC pump and therefore feasible with limited investment for most laboratories who already own nano-LC equipment. At $900 \mathrm{~nL} / \mathrm{min}$, the pump used operates at the absolute lower limits of its specifications, and can only be used in isocratic mode. Also, it is mandatory to keep a high backpressure on the channel at all times because the pump is

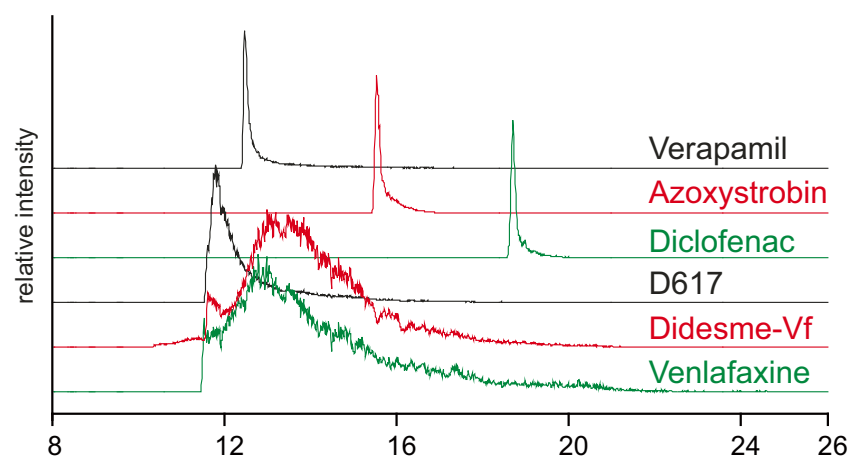

Fig. 2 Online SPE-LC-MS in conventional trap-and-elute (left) and refocusing (right) setup. Extracted ion chromatograms of $[\mathrm{M}+\mathrm{H}]+$ with $10 \mathrm{ppm}$ mass window. From top to bottom verapamil, not able to build up such a pressure ( $\sim 300$ bar) quickly on demand. To this end, a flow restrictor was created using a capillary packed with $3 \mu \mathrm{m} \mathrm{C18}$ chromatographic particles, which keeps the pump under backpressure when the dilution flow is not going to the analytical column. Former implementations of peak refocusing in narrowbore and analytical scale systems commonly used two pumps for the formation of a gradient, where the organic solvent pump runs over the cartridge [10,32]. Therefore, the cartridge is permanently eluted with methanol during the analytical run. In the nano-LC system shown here, a gradient can only be formed by the nanoLC pump; therefore, the elution phase must be separated completely from the gradient chromatography phase.

Online SPE Sorbents used in offline SPE are often available in large particle sizes $(30-100 \mu \mathrm{m})$. However, in a miniaturized online system where the cartridge inner diameter is only $130 \mu \mathrm{m}$, only chromatography grade material (particle sizes $\sim 5 \mu \mathrm{m}$ ) can be used. Oasis HLB (Waters, USA), a broad-range SPE adsorbent commonly used in environmental chemistry based on vinylpyrrolidone-divinylbenzene copolymer, is available in $5 \mu \mathrm{m}$ particle size, and was chosen as the basis material for extraction. In both online and offline SPE applications, good results were obtained by using a mixture of sorbents including Oasis HLB, anion and cation exchange sorbents, and Isolute ENV+ (Biotage, Uppsala, SE), a polymeric material which typically shows higher retention for polar analytes [33]. In an attempt to reproduce the broad-range selectivity of the mixed material cartridges, different combinations of materials in the SPE cartridge were screened preliminarily with 45 substances at a concentration of $100 \mathrm{ng} / \mathrm{L}$. The selected substance range included species which are cationic (14) and anionic (3) or exhibit multiple charges (4) at the loading $\mathrm{pH}$ of 7. Therefore combinations of Oasis HLB with weak cation and anion exchangers (PolyCAT and PolyWAX, PolyLC, USA) in $5 \mu \mathrm{m}$ particle size, with a single cartridge or two cartridges in series were tested. During loading at $\mathrm{pH} 7$, both ion exchangers are in charged state. During elution under acidic conditions, WCX and potential anions will be

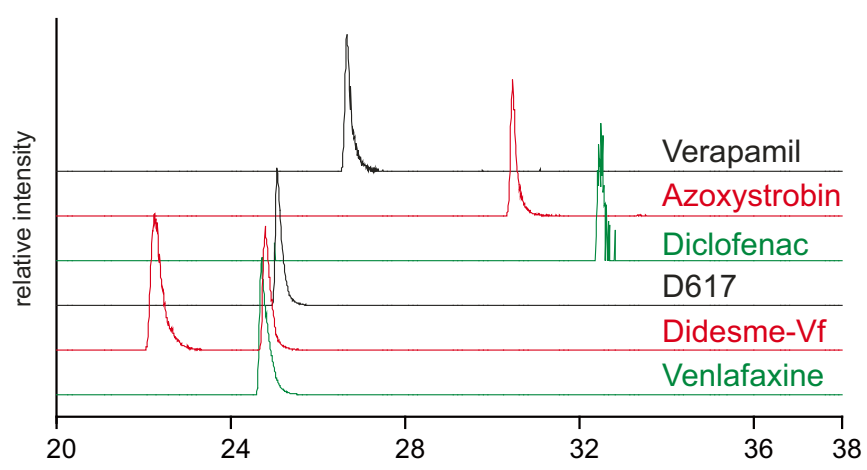

azoxystrobin, diclofenac, D617 verapamil metabolite, $\mathrm{N}, \mathrm{N}$ - and $\mathrm{N}, \mathrm{O}$ didesmethylvenlafaxine (Didesme-Vf), venlafaxine 
uncharged, whereas WAX and potential cations will stay charged, thus disrupting both types of ionic interactions, such that a single elution condition is sufficient for any combination of sorbents. However, only minor differences in selectivities between the different cartridge types were observed. The fact that the system is to be used for transformation product identification, many of which are anionic, led to the adoption of a HLB/WAX mixture as the final cartridge.

To challenge the final system, the screening was expanded to 59 compounds including very hydrophilic substances. At a concentration of $50 \mathrm{ng} / \mathrm{L} 41$ compounds were retained sufficiently for subsequent validation (see ESM Table S5). The remaining 18 compounds could either not be recovered at all, or showed very weak peaks suggesting that only a fraction of the compound was retained on the cartridge. As shown in Fig. S4 in the ESM, the unretained analytes were all in the highly polar range. Recovery was particularly poor for hydroxylated metabolites of pesticides and pharmaceuticals such as atrazine-2-hydroxy and terbuthylazine-2-hydroxy, which are often reported to be not well retained on SPE cartridges. Of the seven non-recovered analytes with log Dow $(\mathrm{pH} 7)>0.5$, six were hydroxylated metabolites. This shows an obvious limitation of the chosen SPE sorbent system. Combination of the currently used sorbents with stronger hydrophilic SPE materials would likely improve the range of accessible analytes; however, at the time of the study, such sorbents were not available in suitable particle size.

To examine the elution properties and the capacity of the SPE system, analyte recovery was tested with both a single cartridge and two cartridges in series. An elution duration of 9 min proved suitable for the elution of the targeted analytes for both cases.

Backflush (shown in Fig. 1) and forward elution (see ESM Fig. S3) setups were tested. It is often recommended to set up nano-LC systems in forward elution mode to reduce the risk of clogging [34]. However, backflush elution was selected in this case, since we observed less carryover and better elution of strongly retained analytes (e.g., Diclofenac) in backflush elution. This is likely related to the fact that strongly retained analytes travel a shorter distance in the cartridge during trapping; therefore, in backflush elution they have a shorter elution pathway and can be eluted completely, while in forward elution they might not be fully eluted because of a longer elution pathway. Enrichment flow rates from 2.5 to $10 \mu \mathrm{L} / \mathrm{min}$ were tested, however, no marked influence of flow rate on extraction recovery was noted. Ten microliters per minute was chosen as enrichment flow rate to keep total runtime short. Likely, a drop in efficiency could have been observed at even higher flow rates. However, the maximal flow rate is limited not by the cartridge itself, but by the backpressure generated by the $20 \mu \mathrm{m}$ ID capillary which follows the trap.
Chromatography Figure S5 in the ESM shows chromatographic profiles of the quantified analytes in the final method at the validation level of $50 \mathrm{ng} / \mathrm{L}$, including peak width at halfmaximum, $10 \%$, and $5 \%$. To evaluate the chromatographic performance, peak tailing factor and peak asymmetry, which both describe the chromatographic suitability of a peak in terms of tailing, were computed (see ESM, "Materials and methods") and are shown in ESM Table S2. For comparison, peak width and tailing/asymmetry were also calculated for an established LC-HRMS/MS method [35]. For the same substances, peak widths were in general equal or narrower than in the established method, showing the chromatographic competitiveness of the method. The median of the tailing factors is 1.45 , and $90 \%$ are below 1.65. The compared LC-HRMS/MS method performs slightly better (median 1.23, $90 \%$ below 1.46); the slight tailing is likely a consequence of the transfer from SPE cartridge to analytical column [36]. However, all tailing factors are below 2, which is a typical requirement for routine analysis [37] and is unproblematic for quantification.

Extraction recovery The method was finally validated with a set of 41 substances. An overview of the results is presented in Table 2. The observed extraction recoveries for all substances cover a wide range from $<10 \%$ to complete extraction. The determination of extraction recovery required collection of the eluate from an online SPE run and subsequent reinjection on the online SPE system after addition of an internal standard. Since all compounds therefore underwent extraction losses twice, some extraction recoveries for weakly retained compounds could not be quantified. However, it is notable that even for analytes with very low recoveries, detection limits in the low nanogram per liter range were reached (see below, e.g., Trifloxystrobin). This reinforces the finding that nano-LC in combination with nano-ESI can be used for highly sensitive quantitation of small molecules, and in some cases, the already good detection limits could be further improved by a factor of 10 or more with the use of more efficient SPE sorbents.

Carryover The repeated use of the same extraction cartridge, in combination with strongly sorptive compounds, often contributes to carryover in online SPE system. A thorough washing procedure was instated to minimize cross-contamination (see above). Cross-contaminations could not be completely eliminated, however for the majority of substances the carryover was absent or $<1 \%$. Exceptions with a higher carryover were the insecticide DEET (up to $15 \%$ ), the didesmethylvenlafaxine metabolites $(\mathrm{N}, \mathrm{N}$ - and $\mathrm{N}, \mathrm{O}-, 5-10 \%)$, mefenamic acid (2$5 \%$ ) and metoprolol (1\%). For best quantification results, it is advised to insert a blank run or a shortened blank run between two samples. 
Table 2 Summarized validation results for validated compounds in matrices. $(*)$ : not determinable $(* *)$ : The two compounds are isobaric and coeluting, they are reported as the sum of concentrations

\begin{tabular}{|c|c|c|c|c|c|c|c|c|c|c|c|c|c|c|c|c|}
\hline \multirow[t]{2}{*}{ Compound } & \multicolumn{5}{|c|}{ LOQ in $n g / L$} & \multicolumn{4}{|c|}{$\mathrm{RR}$ in $\%$} & \multicolumn{4}{|c|}{$\mathrm{RSD}$ in $\%$} & \multicolumn{3}{|c|}{$\mathrm{ER}$ in $\%$} \\
\hline & NP & NP MS/MS & SW & GM & $\mathrm{MC}$ & NP & SW & GM & $\mathrm{MC}$ & NP & SW & GM & $\mathrm{MC}$ & SW & GM & $\mathrm{MC}$ \\
\hline $\begin{array}{l}\text { 10-11-Dihydro- } \\
\text { carbamazepine }\end{array}$ & 0.1 & 0.1 & 0.1 & 0.2 & 0.2 & 113 & 113 & 99 & 118 & 9 & 9 & 8 & 10 & 93 & 90 & 77 \\
\hline $\begin{array}{l}\text { 4/5-Methyl-1H- } \\
\text { benzotriazole }(* *)\end{array}$ & 5 & 5 & 8.2 & 8.9 & 7.4 & 106 & 97 & 127 & 105 & 9 & 14 & 15 & 3 & 43 & 41 & 16 \\
\hline Atrazine & 0.1 & 0.1 & 0.1 & 0.2 & 0.1 & 120 & 104 & 103 & 104 & 15 & 3 & 4 & 2 & 88 & 107 & 76 \\
\hline Atrazine-desethyl & 5 & 5 & 5.2 & 8.1 & 10.6 & 86 & 93 & 103 & 99 & 9 & 4 & 5 & 2 & 26 & 16 & 4 \\
\hline $\begin{array}{l}\text { Atrazine-desethyl- } \\
\text { desisopropyl }\end{array}$ & 2 & 5 & 2.9 & 4 & 3.6 & 107 & 86 & 71 & 105 & 18 & 10 & 5 & 20 & 108 & 84 & 47 \\
\hline Atrazine-desisopropyl & 5 & 20 & 6.4 & 8.7 & 9.9 & 120 & 74 & 69 & 73 & 13 & 10 & 6 & 12 & 124 & 93 & 59 \\
\hline Azoxystrobin & 5 & 20 & 4.4 & 6.4 & 6.4 & 88 & 84 & 76 & 86 & 17 & 7 & 7 & 29 & 74 & 68 & 78 \\
\hline Azoxystrobin-acid & 5 & 5 & 5.7 & 8.5 & 6.9 & 85 & 86 & 78 & 91 & 8 & 15 & 3 & 11 & 70 & 55 & 54 \\
\hline Bezafibrate & 5 & 20 & 7.2 & 13.3 & 23.4 & 90 & 96 & 90 & 0 & 3 & 13 & 11 & $(*)$ & 38 & 15 & 11 \\
\hline Carbamazepine & 0.1 & 0.2 & 0.2 & 0.1 & 0.2 & 104 & 125 & 105 & 108 & 5 & 13 & 5 & 6 & 106 & 104 & 58 \\
\hline $\begin{array}{l}\text { Carbamazepine-10-11- } \\
\text { epoxide }\end{array}$ & 1 & 5 & 1.5 & 1.3 & 1.6 & 90 & 100 & 98 & 105 & 2 & 4 & 4 & 9 & 29 & 38 & 10 \\
\hline Carbendazim & 20 & 20 & 16.9 & 22 & 28.6 & 97 & 85 & 88 & 91 & 2 & 4 & 4 & 2 & 36 & 37 & 22 \\
\hline Clarithromycin & 2 & 5 & 3.3 & 2.5 & 17.5 & 91 & 245 & 265 & 69 & 25 & 2 & 3 & 65 & 7 & 3 & 30 \\
\hline Cyproconazole & 0.5 & 1 & 0.5 & 0.6 & 0.3 & 110 & 81 & 80 & 84 & 57 & 7 & 6 & 27 & 114 & 101 & 79 \\
\hline D617 & 5 & 5 & 4.4 & 5.6 & 4.7 & 69 & 101 & 106 & 95 & 22 & 9 & 5 & 4 & 82 & 52 & 73 \\
\hline DEET & 20 & 20 & 27.8 & 30.3 & 30.9 & 106 & 98 & 106 & 108 & 18 & 16 & 6 & 24 & $(*)$ & $(*)$ & $(*)$ \\
\hline Diclofenac & 10 & 10 & 14.6 & 25.1 & 142.9 & 93 & 93 & 97 & 0 & 19 & 2 & 7 & 0 & 86 & 114 & 71 \\
\hline Epoxyconazole & 0.2 & 1 & 0.2 & 0.3 & 0.1 & 101 & 102 & 100 & 86 & 19 & 3 & 6 & 16 & 64 & 85 & 73 \\
\hline Fluconazole & 20 & 20 & 8.6 & 28.9 & 126.6 & 101 & 82 & 113 & 205 & 18 & 4 & 11 & 4 & $(*)$ & $(*)$ & $(*)$ \\
\hline Irgarol & 0.1 & 0.2 & 0.1 & 0.2 & 0.2 & 93 & 93 & 86 & 80 & 13 & 5 & 1 & 5 & 84 & 90 & 69 \\
\hline Irgarol-descyclopropyl & 2 & 2 & 2.4 & 3.3 & 3.4 & 79 & 90 & 112 & 121 & 32 & 10 & 10 & 3 & 59 & 110 & 65 \\
\hline Kresoxim-methyl & 5 & 5 & 8.1 & 26.7 & 10.1 & 114 & 78 & 0 & 48 & 11 & 1 & $(*)$ & 7 & 86 & 42 & 95 \\
\hline Mefenamic acid & 0.5 & 1 & 0.3 & 0.5 & 0.3 & 96 & 96 & 102 & 127 & 4 & 5 & 3 & 41 & 66 & 62 & 32 \\
\hline Metoprolol & 20 & 20 & 24.1 & 22.1 & 29.3 & 96 & 110 & 100 & 97 & 2 & 2 & 3 & 4 & 70 & 68 & 49 \\
\hline $\begin{array}{l}N \text {-Desmethyl- } \\
\text { clarithromycin }\end{array}$ & 5 & 5 & 2.9 & 3.7 & 5.9 & 94 & 141 & 144 & 48 & 2 & 2 & 4 & 173 & 13 & 6 & 28 \\
\hline $\begin{array}{l}N \text {-Desmethyl- } \\
\text { venlafaxine }\end{array}$ & 0.2 & 1 & 0.2 & 0.3 & 0.2 & 98 & 121 & 105 & 99 & 1 & 2 & 6 & 4 & 89 & 74 & 59 \\
\hline $\begin{array}{l}N, N \text {-Didesmethyl- } \\
\text { venlafaxine }\end{array}$ & 0.5 & 0.5 & 0.6 & 0.6 & 0.6 & 91 & 93 & 105 & 100 & 10 & 9 & 9 & 9 & 80 & 53 & 58 \\
\hline $\begin{array}{l}N, O \text {-Didesmethyl- } \\
\text { venlafaxine }\end{array}$ & 5 & 5 & 2.5 & 4.2 & 9.2 & 97 & 100 & 86 & 95 & 7 & 7 & 10 & 3 & 15 & 46 & 40 \\
\hline $\begin{array}{l}O \text {-Desmethyl- } \\
\text { venlafaxine/ } \\
\text { Tramadol }(* *)\end{array}$ & 1 & 1 & 0.9 & 1.1 & 1.6 & 96 & 109 & 101 & 101 & 1 & 2 & 4 & 5 & $(*)$ & 49 & 40 \\
\hline Propiconazole & 0.5 & 1 & 0.7 & 0.7 & 0.4 & 95 & 98 & 94 & 89 & 5 & 3 & 4 & 6 & 68 & 82 & 60 \\
\hline Tebuconazole & 0.5 & 2 & 0.7 & 0.7 & 0.4 & 92 & 93 & 92 & 107 & 4 & 5 & 4 & 14 & 75 & 77 & 70 \\
\hline Terbuthylazine & 5 & 5 & 4.6 & 6.1 & 5.9 & 108 & 106 & 101 & 106 & 39 & 13 & 8 & 5 & 100 & 86 & 70 \\
\hline $\begin{array}{l}\text { Terbuthylazine- } \\
\text { 2-OH }\end{array}$ & 5 & 20 & 3.3 & 7.6 & 9.4 & 88 & 67 & 108 & 113 & 10 & 7 & 4 & 15 & 25 & 26 & 9 \\
\hline $\begin{array}{l}\text { Terbuthylazine- } \\
\text { desethyl }\end{array}$ & 0.5 & 0.5 & 0.6 & 0.7 & 0.7 & 101 & 110 & 102 & 112 & 0 & 9 & 6 & 10 & 102 & 83 & 52 \\
\hline Terbutryn & 0.1 & 0.1 & 0.1 & 0.1 & 0.2 & 99 & 105 & 93 & 104 & 4 & 2 & 5 & 2 & 88 & 98 & 83 \\
\hline Tramadol $N$-oxide & 20 & 20 & 23.4 & 31.5 & 52.1 & 167 & 72 & 60 & 33 & 80 & 5 & 16 & 100 & $(*)$ & $(*)$ & $(*)$ \\
\hline Trifloxystrobin & 2 & 5 & 2.5 & 7.3 & 1.8 & 111 & 109 & 53 & 126 & 28 & 12 & 8 & 30 & 14 & 8 & 31 \\
\hline Venlafaxine & 0.5 & 1 & 0.6 & 0.6 & 0.6 & 95 & 120 & 112 & 105 & 4 & 4 & 8 & 6 & 81 & 69 & 59 \\
\hline Verapamil & 5 & 20 & 5.6 & 3.7 & 0.6 & 84 & 108 & 112 & 88 & 22 & 2 & 4 & 6 & $(*)$ & $(*)$ & $(*)$ \\
\hline
\end{tabular}

$R R$ relative recovery, $R S D$ relative standard deviation, $E R$ extraction recovery 
Sensitivity LOQs in NPW are reported as MS LOQs (where the chromatographic peak in MS reaches acceptance criteria) and MS/MS LOQs (the first concentration where a confirming fragment was observed). For all validated analytes, LOQs of less than $20 \mathrm{ng} / \mathrm{L}$ in NPW were obtained, while 14 compounds reached sub-ng/L quantification limits. The median LOQ is at $2 \mathrm{ng} / \mathrm{L}$. A comparison of the different matrices is shown in Fig. 3. In surface water and growth medium, moderate matrix effects are observed; in both cases, 14 compounds are quantifiable in sub-nanogram per liter concentrations, whereas the highest LOQs are 28 and $31 \mathrm{ng} / \mathrm{L}$ and the median LOQs are 2.9 and $3.7 \mathrm{ng} / \mathrm{L}$, respectively, still representing very high sensitivity. In Microcystis lysate, matrix effects were most clearly manifested. Fourteen analytes still exhibit subnanogram per liter sensitivity, and median LOQ is $3.6 \mathrm{ng} / \mathrm{L}$, however, specific analytes (tramadol N-oxide, LOQ $52 \mathrm{ng} / \mathrm{L}$; fluconazole, $127 \mathrm{ng} / \mathrm{L}$; diclofenac, $143 \mathrm{ng} / \mathrm{L}$ ) were strongly affected. For diclofenac, this may possibly be caused by a coeluting matrix component observed at high intensity in cell lysate $(\mathrm{m} / \mathrm{z} 535.2148)$ which dominates the TIC at the corresponding retention time. The SPE extraction recovery is not affected. For fluconazole, the extraction recovery is low in all matrices, but cannot be excluded as a possible cause for low sensitivity in cell lysate. Detection limits in MC matrix are given in $\mathrm{ng} / \mathrm{L}$ for comparison with other matrices. Since the phytoplankton biomass used in the validation study was $0.3 \mathrm{~g}$ dry weight/L, the corresponding detection limits in Microcystis ranged from 0.33 to $476 \mathrm{ng} / \mathrm{g}$ dry weight.

Precision and relative recovery Figure 4 summarizes precision and relative recovery results over the examined matrices, separating compounds with a matching internal standard from compounds which were quantified using a non-matching internal standard. It should be noted that, when using a non-
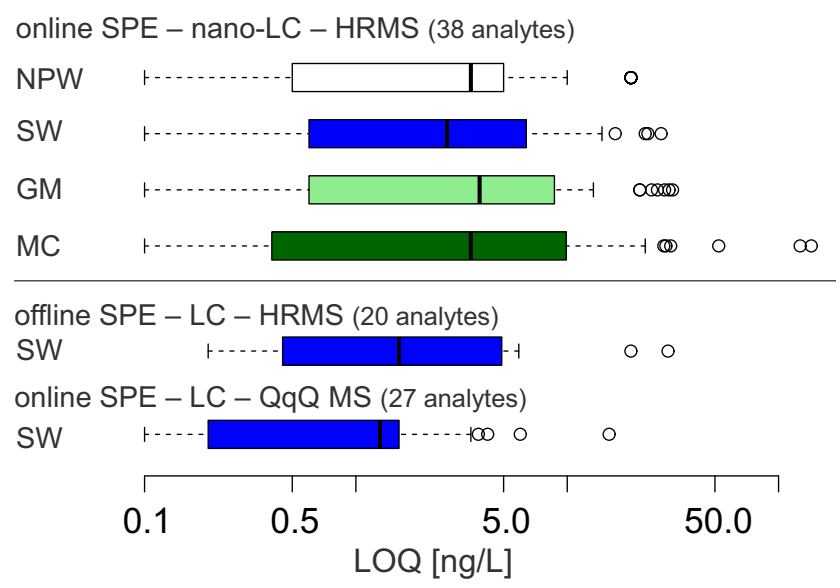

Fig. 3 Top Distribution of limits of quantification in different matrices. $N P W$ nanopure water; $S W$ surface water; $G M$ growth medium; $M C$ Microcystis lysate. Bottom Comparison to existing methods for surface water. Quantification limits for a subset of 20 (offline) or 27 (online) of the analyzed substances with offline SPE-LC-HRMS/MS (Singer et al. [35]) or online SPE-LC-MS/MS (Huntscha et al. [10]) matching internal standard, relative recoveries in different matrices will not necessarily be close to $100 \%$. If the matrix effect is constant, quantification can still be highly accurate by taking the relative recovery into account. The data in the figure exclude clarithromycin, which showed strong matrix interferences in all cases (see Table 2) even though a matching internal standard was used, and could not be quantified satisfactorily. The data shows that relative recovery was generally in the $80-120 \%$ range typically required for quantification in environmental analytics, and precision was generally $<20 \%$, in many cases $<10 \%$. As expected, it was clearly observed that the use of matching internal standards markedly increased the method precision and repeatability. This influence was much stronger for more complex matrices, as seen by the loss in precision for MC matrix. With regards to the use of nanoESI, the use of matching internal standards becomes even more important because nano-ESI fluctuates more strongly depending on gradient conditions, compared to standard flow ESI [38]. Retention time differences between the analyte and the internal standard will not only change the magnitude of matrix suppression, but will inherently contribute to imprecision because of random differences in the spray conditions. For precise quantification under varying conditions, the use of a matching internal standard is essential. This is true in particular when sample pretreatment is kept minimal and potential matrix components can influence compounds unevenly, as was likely the case in MC matrix.

System performance Attention was paid to keep dead volume low, in particular in low-flow pathways, for example the used Stem Trap cartridge was mounted directly into a valve port, thus minimizing dead volume and avoiding additional junctions which have the potential for imperfect connections. A recurring problem in nano-LC is the clogging of small-ID capillaries and columns due to particles in a sample. Therefore, not only filtered solvents and samples were used but additionally an inline filter (Stem Filter, $0.2 \mu \mathrm{m}$ ) was inserted into the valve after the loading pump port, such that the sample was additionally filtered during extraction.

SPE cartridges were observed to be highly durable; they were able to withstand $>100$ injections without observed deterioration. The employed commercial LC column was, however, more prone to clogging. Therefore, an inline filter as the one used for filtering the loading pump flow was also mounted before the HPLC column. However, while the swept volume of the filter is small $(270 \mathrm{~nL})$, it added significant gradient delay and mixing, leading to problems in chromatography and was subsequently removed from the system. While the validation study was carried out without additional column protection, later a commercially available Stem Trap cartridge (130 $\mu \mathrm{m}$ ID, $1.3 \mathrm{~cm}$ length), packed with $3 \mu \mathrm{m} \mathrm{C18}$ particles was inserted into the valve port before the column, acting as a 


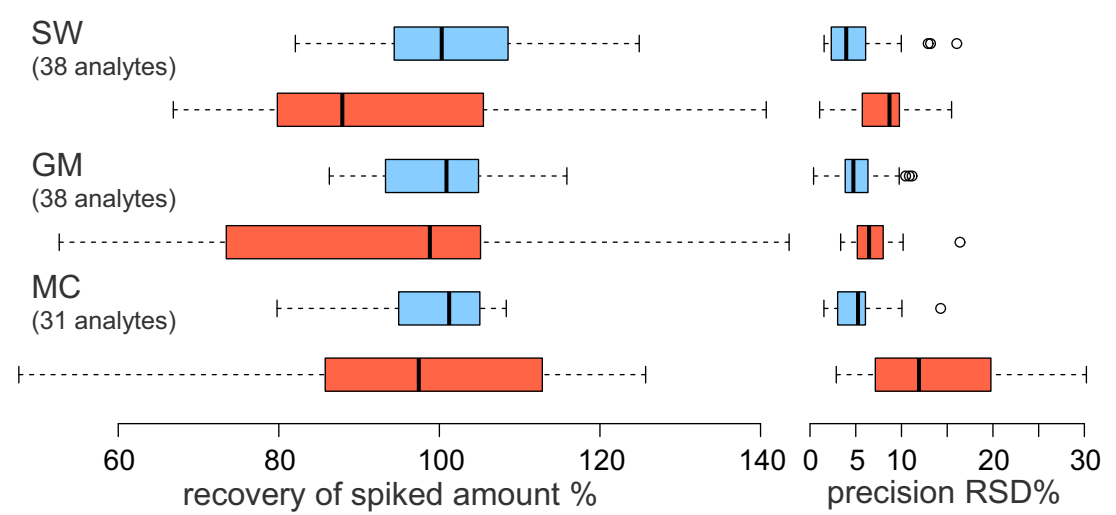

Fig. 4 Relative recovery and precision of all analytes in different matrices, separated by matching ILIS (blue, 21 compounds) and nonmatching ILIS (red, 17 compounds). Data for MC exclude compounds with strong matrix interference (mefenamic acid, DEET, bezafibrate, $N$ desmethylclarithromycin) and with detection limits $>50 \mathrm{ng} / \mathrm{L}$ (fluconazole, diclofenac, tramadol N-oxide) guard column. This approach increased column lifetime markedly.

\begin{abstract}
Application: Bioaccumulation in Microcystis aeruginosa In application to a real-world problem, bioaccumulation of organic micropollutants in Microcystis aeruginosa was determined using a mixture of micropollutants (see SI). Microcystis culture was incubated for $24 \mathrm{~h}$ with a mixture of micropollutants at $100 \mu \mathrm{g} / \mathrm{L}$ concentration, and the cells lysed and analyzed. While most substances accumulated in negligible amounts, high bioconcentration was found for trifloxystrobin $(9.1 \pm 1.2 \mu \mathrm{g} / \mathrm{g} \mathrm{DW})$ and atrazine $(9.3 \pm 2 \mu \mathrm{g} /$ $\mathrm{g}$ DW). In addition, using suspect screening with exact masses and MS/MS interpretation, the putative transformation product trifloxystrobin acid could be identified in Microcystis cells and in the growth medium (see ESM Fig. S5). This laboratory experiment demonstrates one possible application of the method and its versatility, since the high-resolution MS and MS2 data recorded during the quantitation measurement enabled putative identification of a metabolite without additional measurements.
\end{abstract}

Comparison to known approaches For 20 and 27 compounds, respectively, performance results were compared with established offline SPE-LC-HRMS/MS [35, 39] and online SPE-LC-MS/MS [10] methods for surface water analysis. Both methods employed two-layer mixed cartridges with Oasis HLB and a mixture of Isolute $\mathrm{ENV}+$, weak anion and weak cation exchangers. The offline SPE method comprises the enrichment of $500 \mathrm{~mL}$ to a final volume of $1 \mathrm{~mL}$, wherein $20 \mu \mathrm{L}$ was injected (i.e., the injected volume equivalent is $10 \mathrm{~mL}$ ). In the online SPE method, $20 \mathrm{~mL}$ sample are injected and enriched. The methods used $2.1 \mathrm{~mm}$ and $3.0 \mathrm{~mm}$ ID columns for chromatography, respectively. Given that chromatographic dilution scales with the square of column diameter [19], a comparable sensitivity could be expected from 50$100 \mu \mathrm{L}$ on a $100 \mu \mathrm{m}$ ID column. Indeed, as shown in Fig. 3, the detection limits in surface water for analytes present in the offline and normal flow online methods fall in the same range as the values from the developed method. This shows that minimal sample amounts are sufficient to achieve low nanogram per liter detection limits. In some cases (atrazine, carbamazepine, mefenamic acid) the nano-LC method outperformed both online and offline methods. For strongly polar metabolites for which the nano-LC method is less sensitive (e.g., carbendazim), the low extraction recovery likely contributes to the difference.

\section{Conclusions and outlook}

Herein, we demonstrated for the first time a miniaturized approach for automated online-SPE-nano-LC-HRMS analysis which incorporates peak refocusing. The method is applicable in both water and biological matrices. With a small fraction of the sample amount conventionally used $(88 \mu \mathrm{L}$ for water samples, or $26 \mu \mathrm{g}$ Microcystis dry weight), detection limits matching classical large- and medium-volume approaches were reached. Since sample preparation required was minimal, the approach is suitable for automated processing. While the method was primarily developed for the analysis of low-volume, high-throughput laboratory experiments in multi-well plates, it shows promise for other applications, such as biomonitoring in phytoplankton from sub-mg samples - the required phytoplankton sample quantity could be retrieved from less than $1 \mathrm{~L}$ of lake water. Future applications in combination with miniaturized sampling could be envisioned. Through the hyphenation to HRMS, the system could successfully be used for the tentative identification of a transformation product.

While high sensitivity and good accuracy are reached with many important environmental analytes, currently the most polar analytes (in particular, many hydroxylated transformation products) are not accessible to the online SPE method via 
easily available materials. Custom production or modification [40] of existing materials with small particle size would increase the coverage, as it has been shown that the combination of materials in a layered cartridge can provide good coverage of a wide range of substance classes.

Acknowledgments This research was supported by the Swiss National Science Foundation, grant number 315230141190 . We acknowledge Thorsten Schmidt as the external supervisor of Jonas Mechelke during his Masters thesis.

\section{Compliance with ethical standards}

Conflicts of interest The authors declare that they have no conflict of interest.

\section{References}

1. Richardson SD. Environmental mass spectrometry: emerging contaminants and current issues. Anal Chem. 2012;84:747-78. doi:10. 1021/ac202903d.

2. Richardson SD, Ternes TA. Water analysis: emerging contaminants and current issues. Anal Chem. 2014;86:2813-48. doi:10.1021/ ac500508t.

3. Whitehouse C, Dreyer R. Electrospray interface for liquid chromatographs and mass spectrometers. Anal Chem. 1985;57:675-9.

4. Petrovic M, Farré M, Eljarrat E, Díaz-Cruz MS, Barceló D. Environmental analysis: emerging pollutants. In: Liq. Chromatogr. Appl. 2013;89-410.

5. Huerta B, Rodríguez-Mozaz S, Barceló D. Pharmaceuticals in biota in the aquatic environment: analytical methods and environmental implications. Anal Bioanal Chem. 2012;404:2611-24. doi:10. 1007/s00216-012-6144-y.

6. Andreu V, Picó Y. Determination of currently used pesticides in biota. Anal Bioanal Chem. 2012;404:2659-81. doi:10.1007/ s00216-012-6331-x

7. Bijlsma L, Beltrán E, Boix C, Sancho JV, Hernández F. Improvements in analytical methodology for the determination of frequently consumed illicit drugs in urban wastewater. Anal Bioanal Chem. 2014;406:4261-72. doi:10.1007/s00216-0147818-4.

8. Rogeberg M, Malerod H, Roberg-Larsen H, Aass C, Wilson SR. On-line solid phase extraction-liquid chromatography, with emphasis on modern bioanalysis and miniaturized systems. J Pharm Biomed Anal. 2014;87:120-9. doi:10.1016/j.jpba.2013.05.006.

9. Chen L, Wang H, Zeng Q, Xu Y, Sun L, Xu H, et al. On-line coupling of solid-phase extraction to liquid chromatography-a review. J Chromatogr Sci. 2009;47:614-23. doi:10.1093/ chromsci/47.8.614.

10. Huntscha S, Singer HP, McArdell CS, Frank CE, Hollender J. Multiresidue analysis of 88 polar organic micropollutants in ground, surface and wastewater using online mixed-bed multilayer solid-phase extraction coupled to high performance liquid chromatography-tandem mass spectrometry. J Chromatogr A. 2012;1268:74-83. doi:10.1016/j.chroma.2012.10.032.

11. Togola A, Baran N, Coureau C. Advantages of online SPE coupled with UPLC/MS/MS for determining the fate of pesticides and pharmaceutical compounds. Anal Bioanal Chem. 2014;406:1181-91. doi:10.1007/s00216-013-7248-8.
12. Panditi VR, Batchu SR, Gardinali PR. Online solid-phase extraction-liquid chromatography-electrospray-tandem mass spectrometry determination of multiple classes of antibiotics in environmental and treated waters. Anal Bioanal Chem. 2013;405:5953-64. doi:10. 1007/s00216-013-6863-8.

13. Fayad PB, Prévost M, Sauvé S. On-line solid-phase extraction coupled to liquid chromatography tandem mass spectrometry optimized for the analysis of steroid hormones in urban wastewaters. Talanta. 2013;115:349-60. doi:10.1016/j. talanta.2013.05.038.

14. Jeon J, Kurth D, Hollender J. Biotransformation pathways of biocides and pharmaceuticals in freshwater crustaceans based on structure elucidation of metabolites using high resolution mass spectrometry. Chem Res Toxicol. 2013;26:313-24. doi:10.1021/tx300457f.

15. Hennion MC. Solid-phase extraction: method development, sorbents, and coupling with liquid chromatography. J Chromatogr A. 1999;856:3-54. doi:10.1016/S0021-9673(99)00832-8.

16. Ye X, Kuklenyik Z, Needham LL, Calafat AM. Automated on-line column-switching HPLC-MS/MS method with peak focusing for the determination of nine environmental phenols in urine. Anal Chem. 2005;77:5407-13. doi:10.1021/ac050390d.

17. Gama MR, Collins CH, Bottoli CBG. Nano-liquid chromatography in pharmaceutical and biomedical research. J Chromatogr Sci. 2013;51:694-703. doi:10.1093/chromsci/bmt023.

18. Chervet JP, Ursem M, Salzmann JP. Instrumental requirements for nanoscale liquid chromatography. Anal Chem. 1996;68:1507-12. doi:10.1021/ac9508964.

19. Vissers JPC. Recent developments in microcolumn liquid chromatography. J Chromatogr A. 1999;856:117-43. doi:10.1016/S00219673(99)00692-5.

20. Juraschek R, Dülcks T, Karas M. Nanoelectrospray-more than just a minimized-flow electrospray ionization source. J Am Soc Mass Spectrom. 1999;10:300-8.

21. Shen Y, Moore RJ, Zhao R, Blonder J, Auberry DL, Masselon C, et al. High-efficiency on-line solid-phase extraction coupling to 15$150-\mu \mathrm{m}$-i.d. column liquid chromatography for proteomic analysis. Anal Chem. 2003;75:3596-605.

22. Shen Y, Tolić N, Masselon C, Pasa-Tolić L, Camp DG, Hixson KK, et al. Ultrasensitive proteomics using high-efficiency on-line microSPE-nanoLC-nanoESI MS and MS/MS. Anal Chem. 2004;76: 144-54. doi:10.1021/ac030096q.

23. Holste A, Tholey A, Hung CW, Schaumlöffel D. Nano-highperformance liquid chromatography with online precleaning coupled to inductively coupled plasma mass spectrometry for the analysis of lanthanide-labeled peptides in tryptic protein digests. Anal Chem. 2013;85:3064-70. doi:10.1021/ac303618v.

24. Zhang Y, Fonslow BR, Shan B, Baek M-C, Yates JR. Protein analysis by shotgun/bottom-up proteomics. Chem Rev. 2013;113: 2343-94. doi:10.1021/cr3003533.

25. Wilson SR, Malerød H, Holm A, Molander P, Lundanes E, Greibrokk T. On-line SPE-Nano-LC-Nanospray-MS for rapid and sensitive determination of perfluorooctanoic acid and perfluorooctane sulfonate in river water. J Chromatogr Sci. 2007;45:146-52.

26. Berlioz-Barbier A, Baudot R, Wiest L, Gust M, Garric J, CrenOlivé C, et al. MicroQuEChERS-nanoliquid chromatographynanospray-tandem mass spectrometry for the detection and quantification of trace pharmaceuticals in benthic invertebrates. Talanta. 2015;132:796-802. doi:10.1016/j.talanta.2014.10.030.

27. Berlioz-Barbier A, Buleté A, Faburé J, Garric J, Cren-Olivé C, Vulliet E. Multi-residue analysis of emerging pollutants in benthic invertebrates by modified micro-Quick-Easy-CheapEfficient-Rugged-Safe extraction and nanoliquid chromatography-nanospray-tandem mass spectrometry analysis. J Chromatogr A. 2014;1367:16-32. doi:10.1016/j. chroma.2014.09.044. 
28. David A, Abdul-Sada A, Lange A, Tyler CR, Hill EM. A new approach for plasma (xeno)metabolomics based on solid-phase extraction and nanoflow liquid chromatography-nanoelectrospray ionisation mass spectrometry. J Chromatogr A. 2014;1365:72-85. doi:10.1016/j.chroma.2014.09.001.

29. Chetwynd AJ, David A, Hill EM, Abdul-Sada A. Evaluation of analytical performance and reliability of direct nanoLC-nanoESIhigh resolution mass spectrometry for profiling the (xeno)metabolome. J Mass Spectrom. 2014;49:1063-9. doi:10. 1002/jms.3426.

30. Haun J, Leonhardt J, Portner C, Hetzel T, Tuerk J, Teutenberg T, et al. Online and splitless NanoLC $\times$ CapillaryLC with quadrupole/ time-of-flight mass spectrometric detection for comprehensive screening analysis of complex samples. Anal Chem. 2013;85: 10083-90. doi:10.1021/ac402002m.

31. Krauss M, Singer H, Hollender J. LC-high resolution MS in environmental analysis: from target screening to the identification of unknowns. Anal Bioanal Chem. 2010;397:943-51. doi:10.1007/ s00216-010-3608-9.

32. Singer H, Jaus S, Hanke I, Lück A, Hollender J, Alder AC. Determination of biocides and pesticides by on-line solid phase extraction coupled with mass spectrometry and their behaviour in wastewater and surface water. Environ Pollut. 2010;158:3054-64. doi:10.1016/j.envpol.2010.06.013.

33. Kovalova L, McArdell CS, Hollender J. Challenge of high polarity and low concentrations in analysis of cytostatics and metabolites in wastewater by hydrophilic interaction chromatography/tandem mass spectrometry. J Chromatogr A. 2009;1216:1100-8. doi:10. 1016/j.chroma.2008.12.028.

34. Maier-Rosenkranz J. uLC/NanoLC - optimization and troubleshooting. In: HPLC Made to Meas. A Pract. Handb. Optim. 2006;467-486.

35. Singer H, Huntscha S, Hollender J, Mazacek J. MultikomponentenScreening für den Rhein bei Basel. 2009. http://www.eawag.ch/ fileadmin/Domain 1/Abteilungen/uchem/Analytik/pdf/ Multikomponenten-Screening_fuer_den_Rhein.pdf. Accessed 21 Sept 2015

36. Kuklenyik Z, Ye X, Needham LL, Calafat AM. Automated solidphase extraction approaches for large scale biomonitoring studies. J Chromatogr Sci. 2009;47:12-8.

37. Snyder LR, Kirkland JJ, Dolan JW. Basic concepts and the control of separation, Introd. to Mod. Liq. Chromatogr. Hoboken: Wiley; 2010. p. 19-86.

38. Stewart II, Zhao L, Le Bihan T, Larsen B, Scozzaro S, Figeys D, et al. The reproducible acquisition of comparative liquid chromatography/tandem mass spectrometry data from complex biological samples. Rapid Commun Mass Spectrom. 2004;18:1697710. doi:10.1002/rcm.1538.

39. Kern S, Fenner K, Singer HP, Schwarzenbach RP, Hollender J. Identification of transformation products of organic contaminants in natural waters by computer-aided prediction and high-resolution mass spectrometry. Environ Sci Technol. 2009;43:7039-46.

40. Sun JJ, Fritz JS. Chemically modified resins for solid-phase extraction. J Chromatogr. 1992;590:197-202. 